\section{A novel use of arterial spin labelling MRI to demonstrate focal hypoperfusion in individuals with posterior cortical atrophy: a multimodal imaging study}

\section{INTRODUCTION}

Posterior cortical atrophy (PCA) is a rare neurodegenerative syndrome, typically due to Alzheimer pathology, characterised by impairments in higher-order visual function and other parieto-occipital skills. ${ }^{1}$ MRI measures of atrophy and ${ }^{18}$ F-labelled fluorodeoxyglucose (FDG) positron emission tomography (PET) measures of glucose metabolism typically show posterior cortical deficits broadly mirroring the focal cognitive deficits. ${ }^{1}$ By contrast, amyloid PET studies demonstrate that fibrillar amyloid is widely deposited across the cortex. ${ }^{2}$ Arterial spin labelling (ASL) is an MRI methodology that uses endogenous arterial blood water as a tracer to quantify cerebral blood flow (CBF). ${ }^{3}$ We aimed to assess the ability of ASL to detect patterns of reduced CBF in PCA, and to compare these results with those from other imaging modalities.

\section{METHODS}

Five patients fulfilling clinical diagnostic criteria for $\mathrm{PCA}^{4}{ }^{4}$ and five controls attended for three scanning visits usually on consecutive days. On day 1, MRI scans were acquired on a $3 \mathrm{~T}$ Siemens TIM Trio scanner with a 32-channel phased array head-coil. Sequences included a sagittal three-dimensional (3D) MPRAGE T1weighted volumetric scan (acquisition time $9 \mathrm{~min} 23 \mathrm{~s}, \mathrm{TE} / \mathrm{TR} / \mathrm{TI}=2.9 / 2200 /$ $900 \mathrm{~ms}$, dimensions $256 \times 256 \times 208$, voxel size $1.1 \times 1.1 \times 1.1 \mathrm{~mm})$, and coronal T2 fluid-attenuated inversion recovery $(\mathrm{TE} / \mathrm{TR} / \mathrm{TI}=87 / 9000 / 2500 \mathrm{~ms}$, voxel size $0.9375 \times 0.9375 \times 5 \mathrm{~mm})$. Perfusion data were acquired using pulsed ASL (FAIR Q2TIPS) with an 8-segment, backgroundsuppressed 3D GRASE imaging readout ${ }^{5}$ (acquisition time $6 \mathrm{~min} 40 \mathrm{~s}$, TI1/2=800/ $2000 \mathrm{~ms}$, voxel size $3.8 \times 3.8 \times 4.0 \mathrm{~mm}$, refocusing pulse flip angle $130^{\circ}$, five repetitions). A set of three saturation recovery images $(\mathrm{TR}=1,2,5 \mathrm{~s})$ with the same readout module was also acquired to generate tissue M0 and T1 maps for CBF quantification. On day 2, each participant underwent a $10 \mathrm{~min}$ PET scan $50 \mathrm{~min}$ after an intravenous bolus of $300 \mathrm{MBq}$ ${ }^{18}$ F-florbetapir. On day 3, a 20 min PET scan was acquired $30-35 \mathrm{~min}$ postinjection of $185 \mathrm{MBq}{ }^{18}$ F-FDG. PET scans were performed on a GE Discovery ST PET/CT scanner, with CT scans acquired immediately before each scan for attenuation correction.

T1-weighted images were processed using a Gaussian mixture model optimised through expectation maximisation to produce probabilistic segmentations of grey matter, white matter and cerebrospinal fluid. Regional labels were produced simultaneously from the integration of the template parcellations and the segmentation $^{6}$ to define (1) a reference region (whole cerebellum) for the PET normalisation; and (2) frontal, medial temporal, lateral temporal, parietal, posterior cingulate and occipital grey matter lobar regions which were subsequently corrected for total intracranial volume. ASL images were aligned to the T1-weighted image using a symmetric affine registration algorithm to reduce the effects of any residual motion. ${ }^{7}$ CBF maps were generated by fitting a derivative of the general kinetic model to the ASL data. ${ }^{8}$ Florbetapir scans were rated amyloid-positive/negative on grey-scaled images according to standard guidelines; FDG scans were rated for regional hypometabolism. Standard uptake value ratios were calculated for each grey matter lobar region and as a composite, using whole cerebellum as the reference region.

Neuroimaging data from each patient were compared against the controls using a modified $t$ test developed to conduct single participant comparisons, by treating the control sample as sample statistics rather than as population parameters. ${ }^{9}$ To explore the regional relationships between the different techniques, z-scores for each modality and each lobe were calculated relative to controls, and linear regression was used across all brain regions to compare imaging modalities.

\section{RESULTS}

Participant demographics and neuroimaging data are shown in online supplementary table S1. At the time of scanning, the patients (3 female, 2 male) had a mean $\pm \mathrm{SD}$ age of $59.4 \pm 1.8$ years. Singleparticipant images are shown in the figure 1 , alongside the results from a control participant (male, 59 years old) as a reference. All patients were amyloid-positive, with fibrillar amyloid distributed widely across the cortex. Much more focal, posterior cortical reductions in cerebral blood flow, glucose metabolism and atrophy were seen. While atrophy in posterior regions was present in all patients, on visual assessment it was less pronounced than the metabolic deficits assessed using either FDG-PET or ASL.

Quantification of the regional results for each modality is shown in the online supplementary table S1. Compared with controls, there were consistent and significant reductions in grey matter volume and glucose metabolism in the parietal lobe in all patients, and in the parietal, occipital and lateral temporal lobes in the majority. Cerebral blood flow reductions were seen in the parietal and occipital lobes and posterior cingulate for all patients, reaching significance in three. In all cases amyloid deposition was distributed widely across the cortex, including the frontal lobes. Regression analyses showed no significant relationships between amyloid load and any other biomarker. There was no evidence for a relationship between grey matter volume and CBF $(p=0.4)$, borderline evidence for a relationship between grey matter volume and FDG-PET 


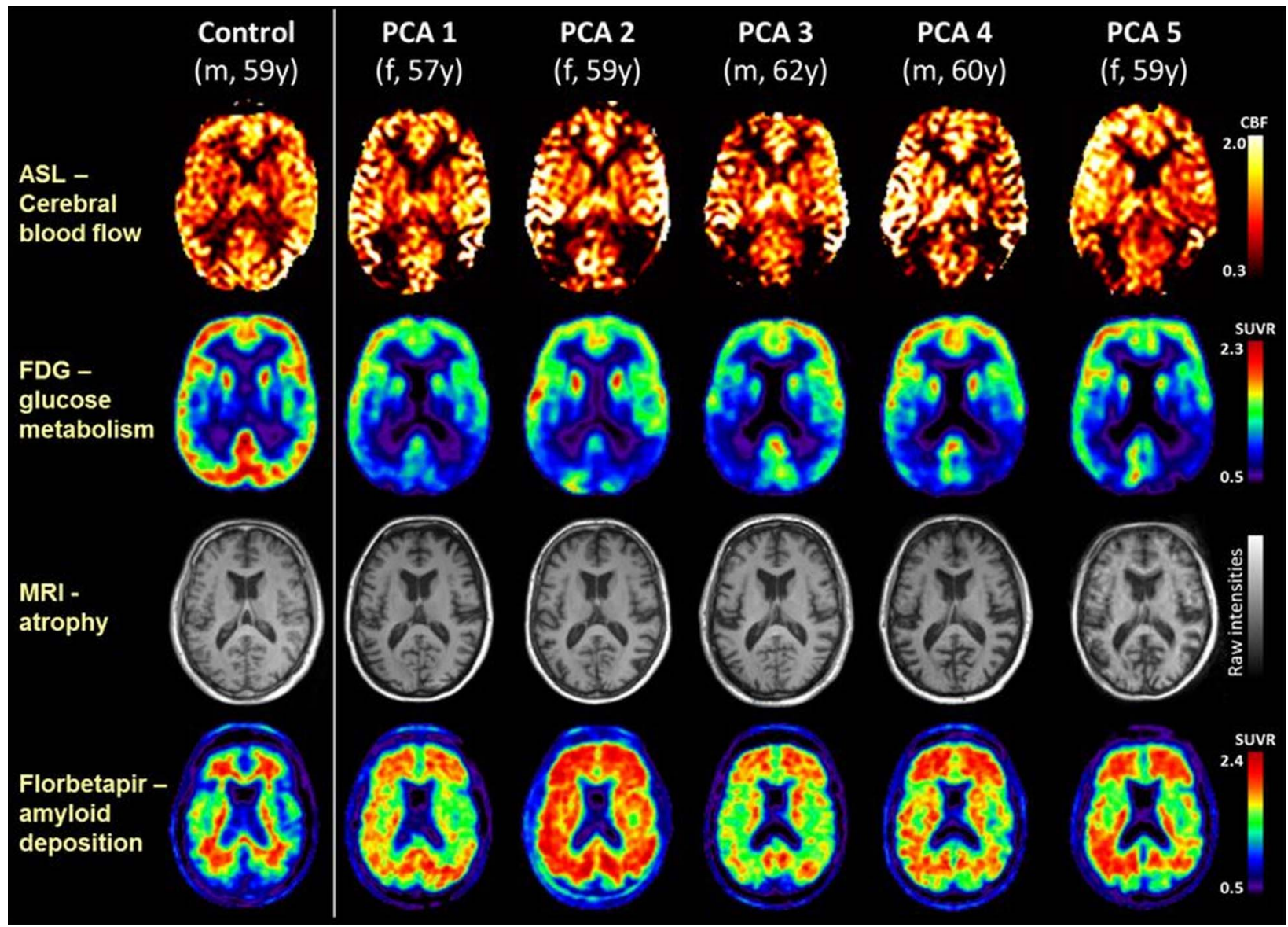

Figure 1 Single-participant axial images for one control participant and five patients with PCA showing cerebral blood flow (ASL), glucose metabolism (FDG-PET), atrophy (structural MRI), and amyloid deposition (florbetapir-PET). For clinical purposes, 18F-florbetapir images should be read on a grey scale. ASL, arterial spin labelling; CBF, cerebral blood flow; FDG-PET, ${ }^{18}$ F-labelled fluorodeoxyglucose positron emission tomography; PCA, posterior cortical atrophy; SUVR, standard uptake value ratio.

metabolism $(p=0.05)$, but a significant correlation between regional $\mathrm{CBF}$ and FDG-PET metabolism $\quad(p=0.004$, $\left.\mathrm{R}^{2}=0.26\right)$

\section{DISCUSSION}

All individuals in this study had clinical and cognitive phenotypes consistent with PCA, and unequivocally positive florbetapir PET scans consistent with underlying $\mathrm{AD}$ pathology. In keeping with previous studies, amyloid deposition was widely distributed across the cortex ${ }^{2}$ while atrophy, hypometabolism and CBF deficits were all more marked in posterior cortical regions.

Previous studies have shown correspondence between the pattern of cerebral hypometabolism measured using FDG-PET and ASL measures of CBF in typical late-onset $\mathrm{AD}^{10}$ and semantic dementia. ${ }^{11}$ Here, we extend these findings to PCA, but importantly show that these group level changes extend to individual patients. Posterior cortical CBF reduction was detectable in each of the individual patients with PCA and broadly mirrored by the observed focal patterns of FDG-PET glucose hypometabolism-the gold standard for detecting regional metabolic changes. Both modalities exhibited more extensive changes than expected purely due to the posterior atrophy seen on structural imaging. These data show that ASL, a non-invasive MR sequence which can implemented on standard clinical 3 T MR scanners, can usefully demonstrate $\mathrm{CBF}$ changes in individual patients with PCA. ASL may be a useful diagnostic adjunct in suspected PCA-and potentially other focal dementias-where an FDG-PET scan is unavailable.

Manja Lehmann ${ }^{1}$ Andrew Melbourne ${ }^{2}$ John C Dickson, ${ }^{3}$ Rebekah M Ahmed, ${ }^{1}$ Marc Modat, ${ }^{2}$ M Jorge Cardoso, ${ }^{2}$

David L Thomas, ${ }^{1,4}$ Enrico De Vita, ${ }^{4,5}$

Sebastian J Crutch, ${ }^{1}$ Jason D Warren, ${ }^{1}$

Colin J Mahoney, ${ }^{1}$ Jamshed Bomanji, ${ }^{3}$

Brian F Hutton, ${ }^{3}$ Nick C Fox, ${ }^{1}$ Xavier Golay, ${ }^{4}$

Sebastien Ourselin, ${ }^{2}$ Jonathan M Schott ${ }^{1}$

${ }^{1}$ Dementia Research Centre, UCL Institute of Neurology, London, UK

${ }^{2}$ Translational Imaging Group, Centre for Medical Image Computing, University College London, London, UK
${ }^{3}$ Institute of Nuclear Medicine, University College London Hospitals, London, UK

${ }^{4}$ Lysholm Department of Neuroradiology, National Hospital for Neurology and Neurosurgery, London, UK ${ }^{5}$ Neuroradiological Academic Unit, Brain Repair \& Rehabilitation, UCL Institute of Neurology, London, UK

Correspondence to Dr Jonathan M Schott, Dementia Research Centre, UCL Institute of Neurology, 8-11 Queen Square, London WC1N 3BG, UK: j.schott@ucl.ac.uk

Acknowledgements The authors acknowledge the support of the Leonard Wolfson Experimental Neurology Centre, the NIHR Queen Square Dementia Biomedical Research Unit and UCL/UCLH Biomedical Research Centre. The Dementia Research Centre is an Alzheimer's Research UK Co-ordinating Centre.

Contributors All the authors were responsible for drafting and revising the manuscript for content. ML, JCD, XG, SO, JMS were responsible for study concept and statistical analysis; ML, AM, JCD, MM, MJC, DLT, $\mathrm{JB}, \mathrm{BFH}, \mathrm{JMS}$ for analysis of the data; RMA, SJC, JDW, $\mathrm{CJM}, \mathrm{NCF}$, and JMS were responsible for patient recruitment; JCD, DLT, EDV for scan acquisition; and JMS for study supervision.

Funding AVID Radiopharmaceuticals (a wholly owned subsidiary of Eli Lilly), and the UK FTD Support Group.

Competing interests $\mathrm{ML}$ is supported by funding from Alzheimer's Research UK (ART-TRFUS2011-2). SC is supported by ESRC/NIHR (ES/K006711/1), EPSRC (EP/M006093/1) and an Alzheimer's Research UK 
Senior Research Fellowship. SO is supported by the EPSRC (EP/H046410/1, EP/J020990/1, EP/K005278), the MRC (MR/J01107X/1), the EU-FP7 (FP7-ICT-20119-601055), the NIHR BRC UCLH/UCL HII (BW.mn. BRC10269). MM and DLT are supported by the UCL Leonard Wolfson Experimental Neurology Centre (PR/ ylr/18575). JMS acknowledges the support of ARUK, the NIHR Biomedical Research Centre, Queen Square Dementia Biomedical Research Unit, and Medical Research Council.

Patient consent Obtained

Ethics approval Queen Square NRES Committee.

Provenance and peer review Not commissioned; externally peer reviewed.

- Additional material is published online only. To view please visit the journal online (http://dx.doi.org/10. 1136/jnnp-2015-312782)

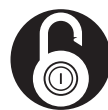

\section{OPEN ACCESS}

Open Access This is an Open Access article distributed in accordance with the terms of the Creative Commons Attribution (CC BY 4.0) license, which permits others to distribute, remix, adapt and build upon this work, for commercial use, provided the original work is properly cited. See: http:// creativecommons.org/licenses/by/4.0/

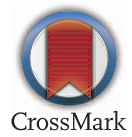

To cite Lehmann M, Melbourne A, Dickson J C, et al. J Neurol Neurosurg Psychiatry 2016;87:1032-1034.

Received 25 November 2015

Accepted 27 November 2015

Published Online First 5 January 2016

J Neurol Neurosurg Psychiatry 2016:87:1032-1034. doi:10.1136/jnnp-2015-312782

\section{REFERENCES}

1 Crutch SJ, Lehmann M, Schott JM, et al. Posterior cortical atrophy. Lancet Neurol 2012;11:170-8.

2 Lehmann M, Ghosh PM, Madison C, et al. Diverging patterns of amyloid deposition and hypometabolism in clinical variants of probable Alzheimer's disease. Brain 2013;136:844-58.

3 Haller S, Zaharchuk G, Thomas D, et al. Arterial spin label perfusion of the brain: emerging clinical applications. Radiology 2015; In Press.

4 Tang-Wai DF, Graff-Radford NR, Boeve BF, et al. Clinical, genetic, and neuropathologic characteristics of posterior cortical atrophy. Neurology 2004;63: 1168-74.

5 Feinberg DA, Ramanna S, Guenther M. Evaluation of new ASL 3D GRASE sequences using parallel imaging, segmented and interleaved k-space at $3 \mathrm{~T}$ with 12- and 32-channel coils. In: Proceedings of the 17th Annual Meeting of ISMRM. Honolulu, HI, USA; 2009:622.

6 Cardoso MJ, Modat M, Wolz R, et al. Geodesic information flows: spatially-variant graphs and their application to segmentation and fusion. IEEE Trans Med Imaging 2015;34:1976-88.

7 Modat M, Cash DM Daga P, et al. Global image registration using a symmetric block-matching approach. J Med Imaging 2014;1:024003.

8 Alsop DC, Detre JA, Golay X, et al. Recommended implementation of arterial spin-labeled perfusion MRI for clinical applications: a consensus of the ISMRM perfusion study group and the European consortium for ASL in dementia. Magn Reson Med 2015;73:102-16.

9 Crawford JR, Garthwaite PH. Investigation of the single case in neuropsychology: confidence limits on the abnormality of test scores and test score differences. Neuropsychologia 2002;40:1196-208.

10 Chen Y, Wolk DA, Reddin JS, et al. Voxel-level comparison of arterial spin-labeled perfusion MRI and FDG-PET in Alzheimer disease. Neurology 2011;77:1977-85.

11 Newberg AB, Wang J, Rao $\mathrm{H}$, et al. Concurrent CBF and CMRGIc changes during human brain activation by combined fMRI-PET scanning. Neuroimage 2005;28:500-6. 